\title{
Higher-Order Spectral Analysis of F-18 Flight Flutter Data
}

\author{
Walter A. Silva* \\ NASA Langley Research Center, Hampton, Virginia, 23681 \\ Shane Dunn ${ }^{\dagger}$ \\ Defence Science and Technology Organisation, Department of Defence, Australia
}

\begin{abstract}
Royal Australian Air Force (RAAF) F/A-18 flight flutter test data is presented and analyzed using various techniques. The data includes high-quality measurements of forced responses and limit cycle oscillation (LCO) phenomena. Standard correlation and power spectral density (PSD) techniques are applied to the data and presented. Novel applications of experimentally-identified impulse responses and higher-order spectral techniques are also applied to the data and presented. The goal of this research is to develop methods that can identify the onset of nonlinear aeroelastic phenomena, such as LCO, during flutter testing.
\end{abstract}

\section{Introduction}

In recent years, there has been a a dramatic increase in the study of nonlinear aeroelastic phenomena. The recent survey paper by Dowell, Edwards, and Strgnac ${ }^{1}$ discusses the broad range of computational and experimental activities aimed at understanding and addressing various types of nonlinear phenomena. This paper, however, will focus on the analysis of data acquired during flight flutter testing of RAAF F/A-18 aircraft, ${ }^{2}$ which includes high-quality measurements of limit cycle oscillation (LCO) phenomena.

During full-scale development of the F/A-18A/B Hornet, it was discovered that when carrying heavy stores on the outboard wing pylons and AIM-9 missiles on the wing tips, the aircraft experiences an "unacceptable $5.0-6.0 \mathrm{~Hz}$ oscillation at low altitude and high speed". ${ }^{3}$ This oscillation is a single-degree-of-freedom LCO.

An aeroelastic LCO is the result of nonlinear dynamics in the structural system or the aerodynamic system, or both. Quite often, as the amplitude of an initial flutter incident increases, the nonlinearities associated with the aeroelastic system become significant. As these nonlinearities grow in magnitude, the aeroelastic system is transformed from an initially unstable linear flutter event into a limited amplitude nonlinear aeroelastic oscillation, or an LCO. Denegri ${ }^{4}$ discusses the different types of LCO encountered by the F-16 aircraft during its flight flutter testing phase.

Recent applications of the Volterra theory to experimental aerodynamics and aeroelasticity are providing valuable knowledge regarding nonlinear aeroelastic behavior. In particular, the experimental identification of aerodynamic impulse responses may provide insight regarding the dominant flow physics of the experiment as well as an automatic data filtering capability. ${ }^{5}$ The application of higher-order spectra (HOS) to flutter data $^{6,7}$ and the identification of Volterra kernels from flight flutter experiments ${ }^{8-10}$ are additional examples of this promising application for the Volterra theory.

The paper is organized as follows. A description of the flight test instrumentation and data acquisition process is presented. This is followed by a description of the various types of data acquired and the data that is analyzed in the paper. A description of higher-order spectral methods is then provided. Finally, the results of the analysis of the data using various techniques is presented and discussed.

\footnotetext{
*Senior Research Scientist, Aeroelasticity Branch, AIAA Associate Fellow.

${ }^{\dagger}$ Head-Aeroelasticity, Air Vehicles Division.
} 


\section{Flight Test Instrumentation and Data Description}

Greater detail on the aircraft configuration and instrumentation for these tests is given in Arms et al ${ }^{11}$ and Keeler et al. ${ }^{12} \mathrm{~A}$ brief summary is given here. The schematic in figure 1 shows the various paths taken by the data. For safety while the aircraft is on-condition, strip charts are monitored to ensure that pre-determined acceleration limits are not exceeded at key locations, and that accelerations do not grow in an unexpected manner during, or following, FECU runs. The FECU is Flutter Exciter Controller Unit and allows the ailerons to be driven in sine dwell, sine sweep or random modes.

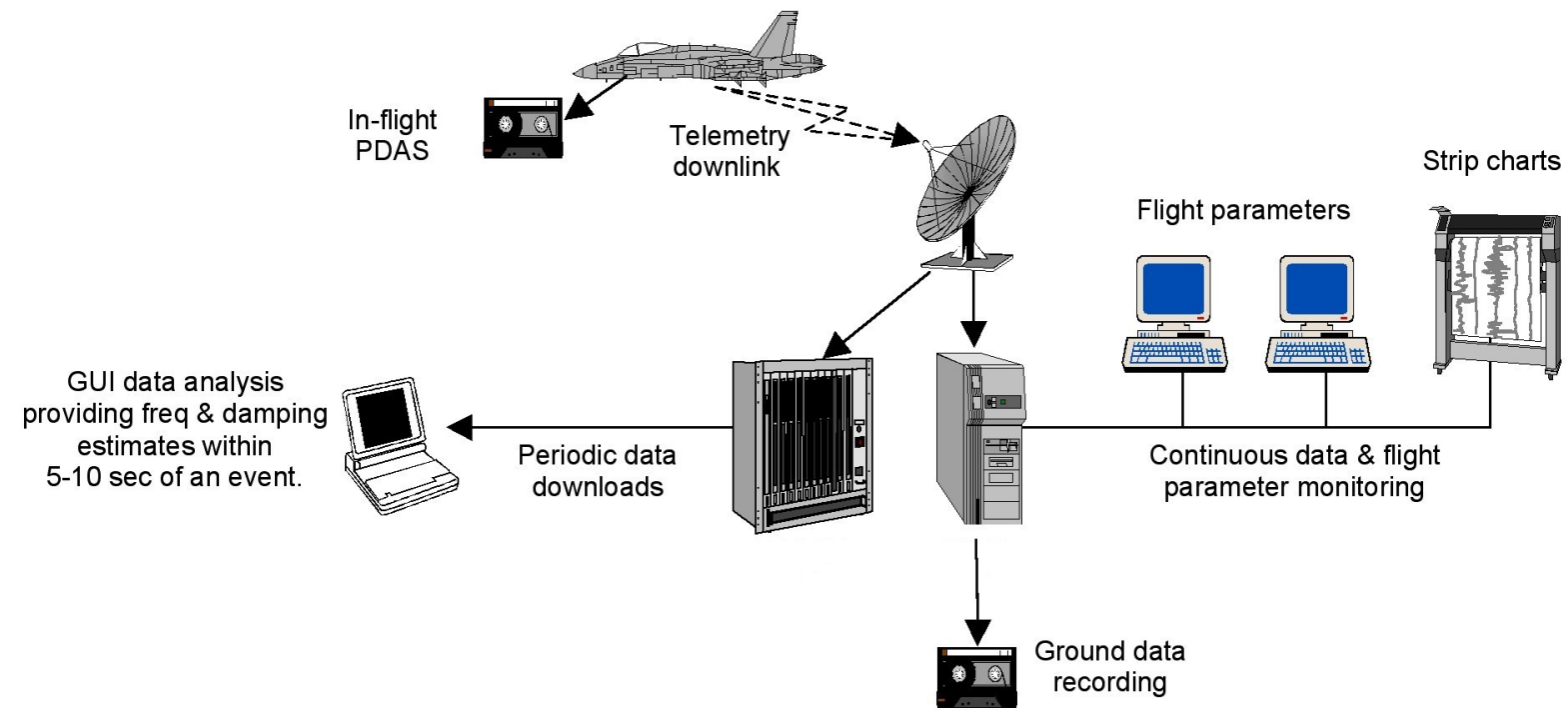

Figure 1. Schematic of the data acquisition process.

The frequencies for the input sine dwells, using the FECU at each stabilized flight test point, were chosen in order to excite the anti-symmetric wing first bending (AW1B) mode. Data from key accelerometers were analyzed and the frequency and damping for the AW1B mode were estimated. In this way, damping estimates were generated so that any dangerous trends could be assessed during the flight. If the damping estimate during a flight dropped to less than 1.5 percent of critical, then any additional planned envelope expansion points at similar or greater Mach no./dynamic pressure would not be attempted. If there was sufficient fuel remaining, other pre-briefed test points at safer parts of the envelope would be flown.

Two MATLAB programs were developed for on-condition and post-flight data analyses. These programs resulted in graphical user interfaces (GUIs) which enabled very fast analysis and visual inspection of the quality of the measured data. The first program analyzed the data in the time-domain. An example of the GUI for this program is shown in figure 2. In figure 2, accelerometer locations also are presented. A number of time domain analysis approaches were available in this program, but the most reliable results were found by examining the decay following a FECU dwell using the Eigensystem Realization Algorithm with Data Correlation (ERA/DC) (Juang et $\mathrm{al}^{13}$ ). For the rare occasions where stick-raps were used, rather than the FECU, random decrement processing $\left(\mathrm{Cole}^{14}\right)$ was used, followed by ERA/DC.

For sweeps using the FECU (typically $2-11 \mathrm{~Hz}$ over $30 \mathrm{sec}$.), a transfer function analysis was performed in the frequency-domain using an output-error curve fit in the complex plane (Ljung ${ }^{15}$ ). This gave an estimate for the modal frequencies and dampings in the frequency range of interest. Such a transfer function analysis, however, requires a reference signal that is linearly proportional to the excitation force. The best reference signals available were aileron rotational position and aileron rotational rate. However, neither of these signals is a true force reference. For this reason, these data were only used in a qualitative sense to provide confirmation of trends found from the analysis of the FECU dwell data. The sweep data were also used to ensure that no instability at an unexpected frequency outside the range of the FECU dwells was approaching.

Between flights, stability margin estimates were generated using the two-degree-of-freedom method of Zimmerman and Weissenburger. ${ }^{16}$ In this case, the two-degree-of-freedom system consisted of the AW1B 


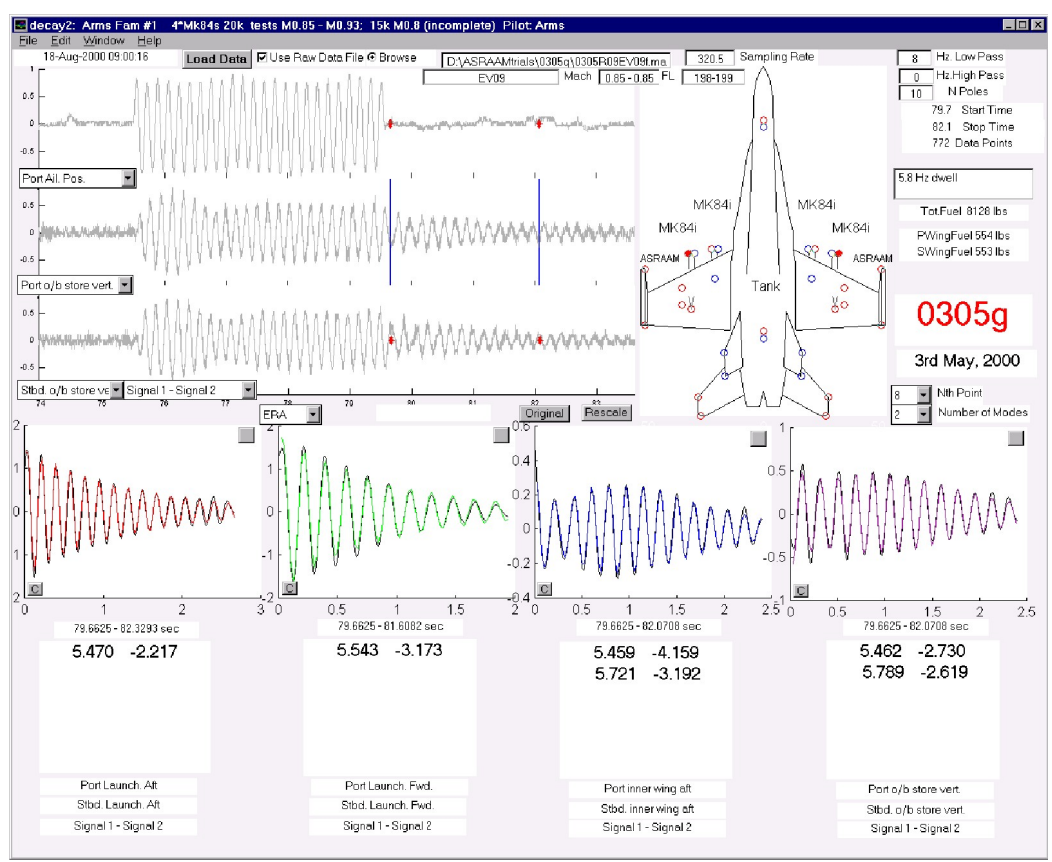

Figure 2. MATLAB Graphical User Interface (GUI) for time-domain analyses of measured data.

and anti-symmetric outboard store pitch (AOBSP) modes. Using this method, extrapolations were made to estimate the altitude at which the system would become unstable. Such stability margin estimates are only valid within regimes where the structural and aerodynamic forces behave linearly; therefore, analysis across test points where there is a significant variation in compressibility and/or shock effects is not valid. For the Mach numbers tested here, it was found that data for $M=0.85$ formed a self consistent set of results, $M=0.9$ another, and data within the range of $\mathrm{M}=0.93$ to $\mathrm{M}=0.95$ gave a third set of results.

Presented in figure 3 is a sample of the type of data acquired during these flight tests. Figure 3 presents aileron sine dwell inputs and resultant accelerations at the aft launcher location during a test run that resulted in a mild flutter condition. As can be seen, the level of positive damping (stable) decreases until negative damping (unstable) is encountered. Quite often during flight flutter testing, LCO phenomena are encountered. These LCO phenomena can mask strong (and dangerous) flutter phenomena which can resurface when the nonlinearity that is creating the LCO disappears. In addition to sine dwell and sine sweep excitations, data acquired during lateral stick raps will also be presented.

The aircraft's AW1B mode exhibits a large decrease in stiffness over a portion of the flight envelope. This nonlinear and temporary reduction in stiffness creates the illusion of a highly damped (i.e., stable) system. But when flying into another portion of the flight envelope, the wing's AW1B mode stiffness returns to its normal (linear) value and the aircraft may encounter an unsafe flutter condition. A contour map of the damping that clearly delineates these low damping $(\mathrm{L})$ and high damping $(\mathrm{H})$ regions of the flight envelope is presented as figure 4 .

Clearly, a strong motivation exists to develop methods that can identify regions of nonlinear behavior prior to their effects becoming significant. With this in mind, the present research effort has focused on the analysis of the flight flutter data using novel spectral analysis methods in addition to the more traditional approaches.

A very large amount of data was acquired during these flight tests. Based on the signal-to-noise ratio of the data and the clearly identified data points, the data can classified as high-quality data. As the intent of this paper is to introduce the application of HOS to flight test data, the results presented are limited to a small subset of the total data. This paper is not intended to be a comprehensive review of the data; some of the references listed above already serve that purpose. In addition, due to the sensitive nature of the data, only generic information (pre-LCO, for example) will be provided instead of the parameters of the flight condition such as Mach number and altitude. 

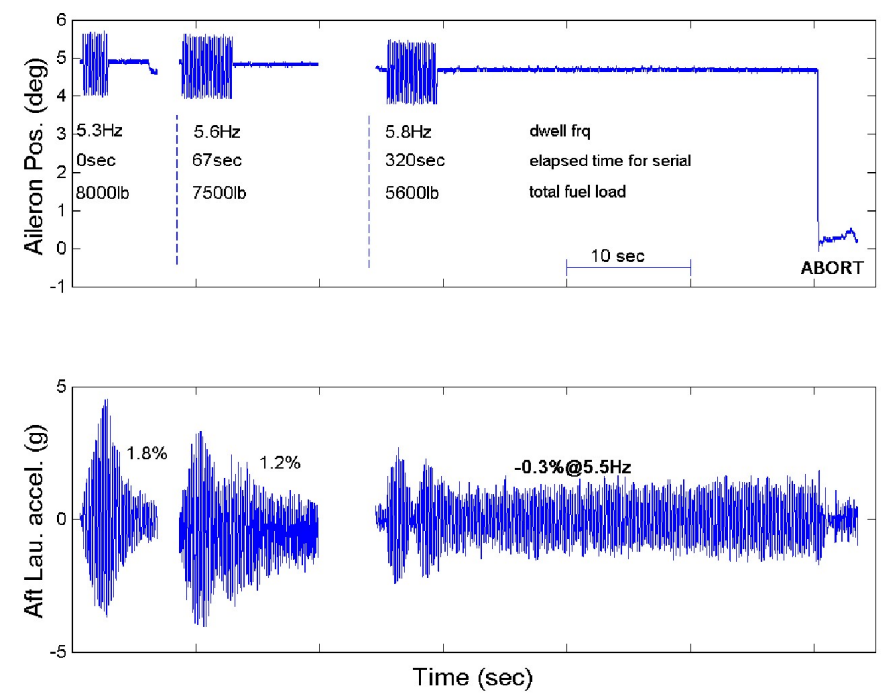

Figure 3. Aileron inputs and resultant accelerations at the aft launcher location for the test run that encountered a mild flutter incident.

\section{H: High Damping L: Low Damping}

4 Aircraft progression when mild flutter was encountered.

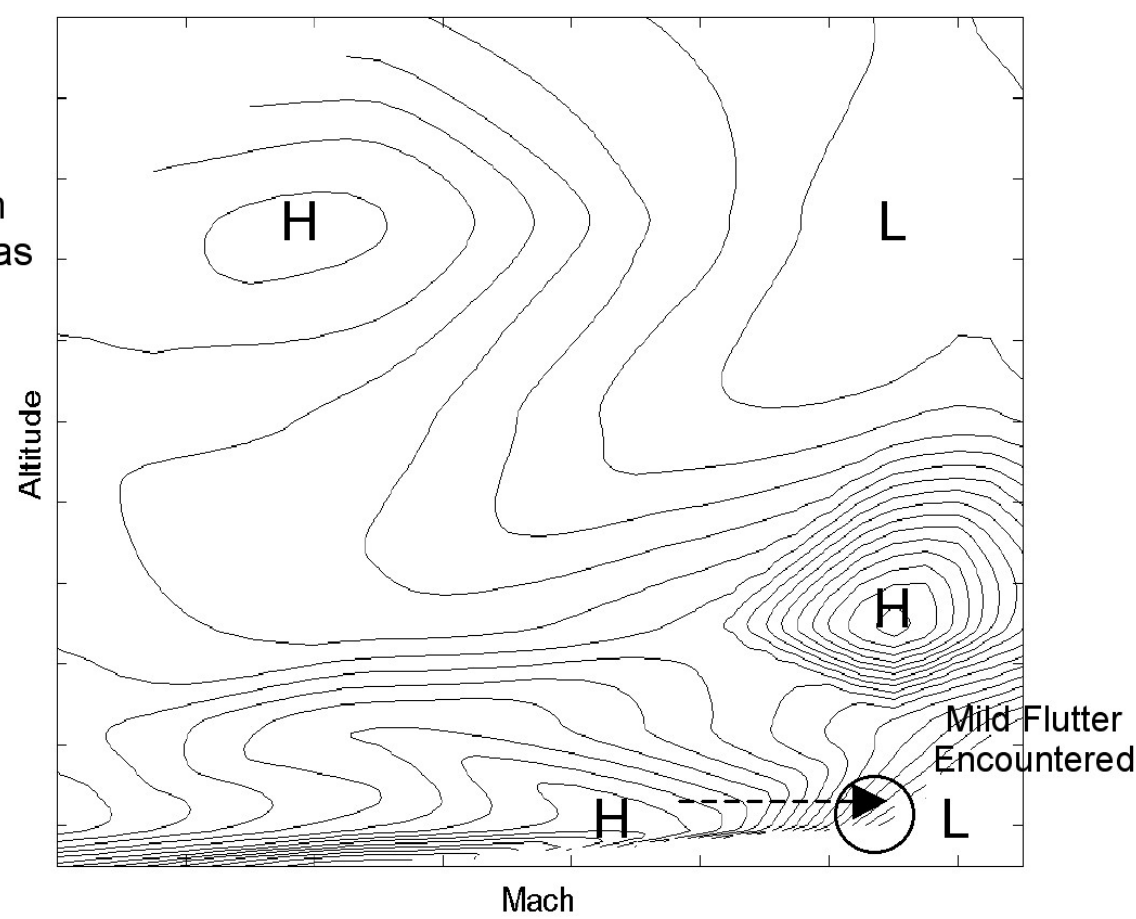

Figure 4. Contours of damping of AW1B mode as a function of Mach number and altitude. 


\section{Higher-Order Spectra}

The primary benefit of higher-order spectra (HOS), also known as higher-order frequency response functions, is that they provide information regarding the interaction of frequencies due to a nonlinear process. For example, bispectra have been used in the study of grid-generated turbulence to identify the nonlinear exchange of energy from one frequency to another (related to the turbulent cascade phenomenon). Linear concepts, by definition, cannot provide this type of information. In addition, higher-order spectra are the frequency-domain version of the Volterra series. For details regarding this relationship, the reader is referred to the recent article by Silva. ${ }^{17}$ Some very interesting and fundamental applications using the frequency-domain Volterra theory ${ }^{18,19}$ and experimental applications of Volterra methods ${ }^{20,21}$ are providing new "windows" on the world of nonlinear aeroelasticity.

In the recent work by Hajj and Silva, ${ }^{6,22}$ the aerodynamic and structural aspects of the flutter phenomenon of a wind-tunnel model are determined via a frequency domain analysis based on a hierarchy of spectral moments. The power spectrum is used to determine the distribution of power among the frequency components in the pressure, strain and acceleration data. The cross-power spectrum, linear coherence, and phase relation of the same frequency components between different signals are used to characterize the bending and torsion characteristics of the model. The nonlinear aspects of the aerodynamic loading are determined from estimates of higher-order spectral moments, namely, the auto- and cross-bispectrum.

For a discrete, stationary, real-valued, zero-mean process, the auto-bispectrum is estimated $a^{23}$

$$
\hat{B}_{x x x}\left[l_{1}, l_{2}\right]=\frac{1}{M} \sum_{k=1}^{M} X_{T}^{(k)}\left[l_{1}+l_{2}\right] X_{T}^{*(k)}\left[l_{1}\right] X_{T}^{*(k)}\left[l_{2}\right]
$$

where $X_{T}^{(k)}[l]$ is the Discrete Fourier Transform of the $k^{t h}$ ensemble of the time series $x(t)$ taken over a time, $T$, and $M$ is the number of these ensembles. The auto-bispectrum of a signal is a two-dimensional function of frequency and is generally complex-valued. In averaging over many ensembles, the magnitude of the auto-bispectrum will be determined by the presence (or absence) of a phase relationship among sets of the frequency components at $l_{1}, l_{2}$, and $l_{1}+l_{2}$. If there is a random phase relationship among these three components, the auto-bispectrum will average to a very small value. Should a phase relationship exist among these frequency components, the corresponding auto-bispectrum will have a large magnitude. ${ }^{24}$ Because a quadratic nonlinear interaction between two frequency components, $l_{1}$ and $l_{2}$, yields a phase relation between them and their summed component, $l_{1}+l_{2}$, the auto-bispectrum can be used to detect a quadratic coupling or interaction among different frequency components of a signal. The level of such coupling in a signal can then be associated with a normalized quantity of the auto-bispectrum, called the auto-bicoherence, and defined as

$$
b_{x x x}^{2}\left[l_{1}, l_{2}\right]=\frac{\left|\hat{B}_{x x x}\left[l_{1}, l_{2}\right]\right|^{2}}{\frac{1}{M} \sum_{k=1}^{M}\left|X_{T}^{(k)}\left[l_{1}\right] X_{T}^{(k)}\left[l_{2}\right]\right|^{2} \frac{1}{M} \sum_{k=1}^{M}\left|X_{T}^{(k)}\left[l_{1}+l_{2}\right]\right|^{2}}
$$

By the Schwarz inequality, the value of $b_{x x x}^{2}\left[l_{1}, l_{2}\right]$ varies between zero and one. If no phase relationship exists among the frequency components at $l_{1}, l_{2}$, and $l_{1}+l_{2}$, the value of the auto-bicoherence will be at or near zero (due to averaging effects). If a phase relationship does exist among the frequency components at $l_{1}, l_{2}$, and $l_{1}+l_{2}$, then the value of the auto-bicoherence will be near unity. Values of the auto-bicoherence between zero and one indicate partial quadratic coupling.

For systems where multiple signals are considered, detection of nonlinearities can be achieved by using the cross-spectral moments. For two signals $x(t)$ and $y(t)$, their cross-bispectrum is estimated as

$$
\hat{B}_{y x x}\left[l_{1}, l_{2}\right]=\frac{1}{M} \sum_{k=1}^{M} Y_{T}^{(k)}\left[l_{1}+l_{2}\right] X_{T}^{*(k)}\left[l_{1}\right] X_{T}^{*(k)}\left[l_{2}\right]
$$

where $X_{T}^{(k)}[l]$ and $Y_{T}^{(k)}[l]$ are the Discrete Fourier Transforms of the $k^{t h}$ ensemble of the time series $x(t)$ and $y(t)$, respectively, over a time, $T$. The cross-bispectrum provides a measure of the nonlinear relationship amongst the frequency components at $l_{1}$ and $l_{2}$ in $x(t)$ and their summed frequency component, $l_{1}+l_{2}$, in $y(t)$. Similar to the auto-bispectrum, the cross-bispectrum of signals $x(t)$ and $y(t)$ is a two-dimensional function in frequency and is generally complex-valued. In averaging over many ensembles, the magnitude 
of the cross-bispectrum will also be determined by the presence, or absence, of a phase relationship among sets of the frequency components at $l_{1}, l_{2}$, and $l_{1}+l_{2}$. If there is a random phase relationship among the three components, the cross-bispectrum will average to a very small value. Should a phase relationship exist amongst these frequency components, the corresponding cross-bispectral value will have a large magnitude. The cross-bispectrum is then able to detect nonlinear phase coupling among different frequency components in two signals because of its phase-preserving effect.

Similarly to defining the auto-bicoherence, one can define a normalized cross-bispectrum to quantify the level of quadratic coupling in two signals. This normalized value is called the cross-bicoherence and is defined as

$$
b_{y x x}^{2}\left[l_{1}, l_{2}\right]=\frac{\left|\hat{B}_{y x x}\left[l_{1}, l_{2}\right]\right|^{2}}{\left.\frac{1}{M} \sum_{k=1}^{M}\left|X_{T}^{(k)}\left[l_{1}\right] X_{T}^{(k)}\left[l_{2}\right]\right|^{2} \frac{1}{M} \sum_{k=1}^{M} Y_{T}^{(k)}\left[l_{1}+l_{2}\right]\right|^{2}}
$$

If no phase relationship exists amongst the frequency components at $l_{1}, l_{2}$ in $x(t)$ and the frequency component at $l_{1}+l_{2}$ in $y(t)$, the value of the cross-bicoherence will be at or near zero. If a phase relationship does exist amongst these frequency components, the value of the cross-bicoherence will be near unity. Values of cross-bicoherence between zero and one indicate partial quadratic coupling. A digital procedure for computing the auto and cross-bicoherence is given by Kim and Powers ${ }^{23}$ and is summarized by Hajj et al. ${ }^{25}$

\section{A. Example}

A simple example is now presented in order to explain some of the concepts associated with HOS. The example ${ }^{26}$ consists of a linear (y) and a nonlinear (z) time series, both with added Gaussian noise (d). The equations for these time series are

$$
\begin{gathered}
x(k)=\sin \left(2 \pi\left(f_{1}\right) k+\frac{\pi}{3}\right)+\sin \left(2 \pi\left(f_{2}\right) k+\frac{\pi}{8}\right) \\
y(k)=x(k)+d(k) \\
z(k)=x(k)+0.05 x^{2}(k)+d(k)
\end{gathered}
$$

where $f_{1}=0.12 \mathrm{~Hz}$ and $f_{2}=0.30 \mathrm{~Hz}$.

Figures 5 and 6 each contain the time series and the associated magnitude of the Fourier transforms for the linear and nonlinear time series, respectively. The dominant frequencies (in this case $0.12 \mathrm{and} 0.30 \mathrm{~Hz}$ ) are clearly visible in both figures. The frequency content for the nonlinear time series indicates the existence of additional frequencies. The nature of these frequencies, whether or not these frequencies are random or the result of a nonlinear coupling process, cannot be discerned from this analysis.

The power spectrum densities (PSD) for the linear and nonlinear time series are presented as figure 7 . Here again, the existence of additional frequencies in the PSD of the nonlinear time series (as compared to the linear time series) is obvious. However, the PSD information cannot be used to discern if these additional frequencies are random or the result of a nonlinear coupling process.

In order to determine if these additional frequencies are random or the result of a nonlinear coupling process, the HOS for these time series must be computed. The magnitude of the bicoherence function for the linear time series, $y$, is presented as figure 8. The two frequency axes correspond to the two frequency indices in Eq. 2. If a significant peak is observed, this implies that the sum of those two frequencies is the result of a quadratic (nonlinear) coupling. It can be seen that the magnitude of the bicoherence function for the linear time series is quite low in value (compared to unity) and that there exist no dominant peaks that would be indicative of a nonlinear coupling.

The magnitude of the bicoherence function for the nonlinear time series is presented as figure 9 . In contrast to the bicoherence function for the linear time series, the bicoherence function for the nonlinear time series has several peaks at or close to unity, indicative of nonlinear (quadratic) coupling at the $\mathrm{x}$ - and $\mathrm{y}$-axis frequencies indicated.

Typically, in order to enhance visualization and interpretation of bicoherence functions, contour plots are viewed. The contour plot for figure 9 is presented as figure 10. The contour plot presents the frequencies that have coupled quadratically to generate the new frequencies that were visible in the PSD functions. 

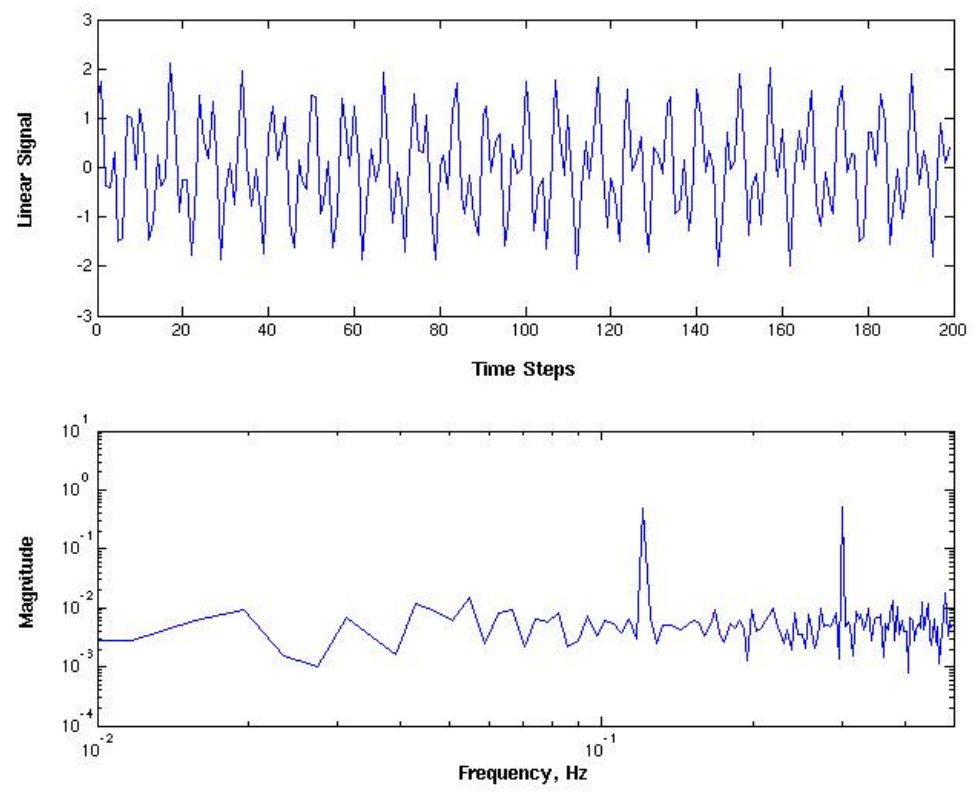

Figure 5. Linear time series and magnitude of frequency response.
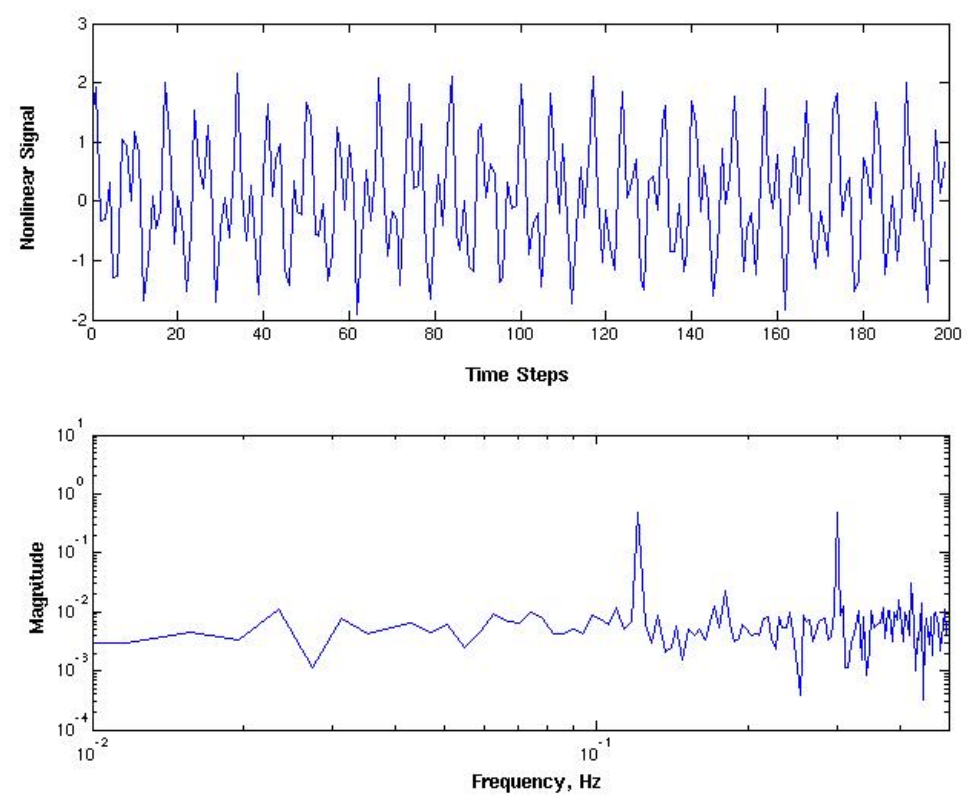

Figure 6. Nonlinear time series and magnitude of frequency response. 

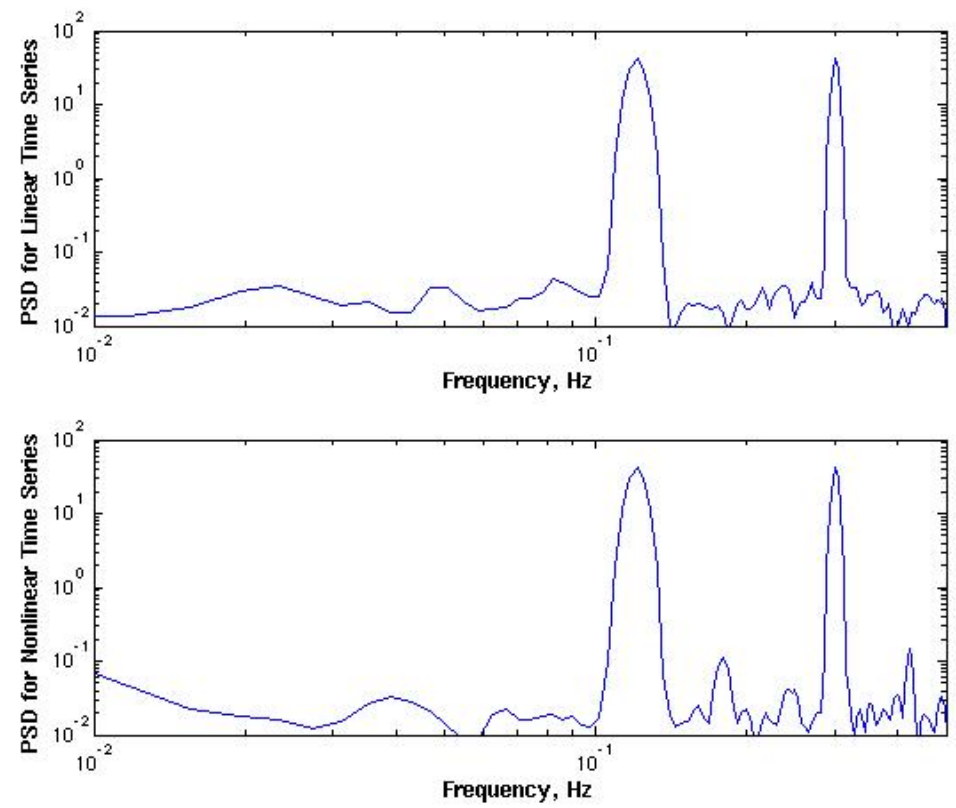

Figure 7. Power spectrum density (PSD) functions for linear and nonlinear time series.

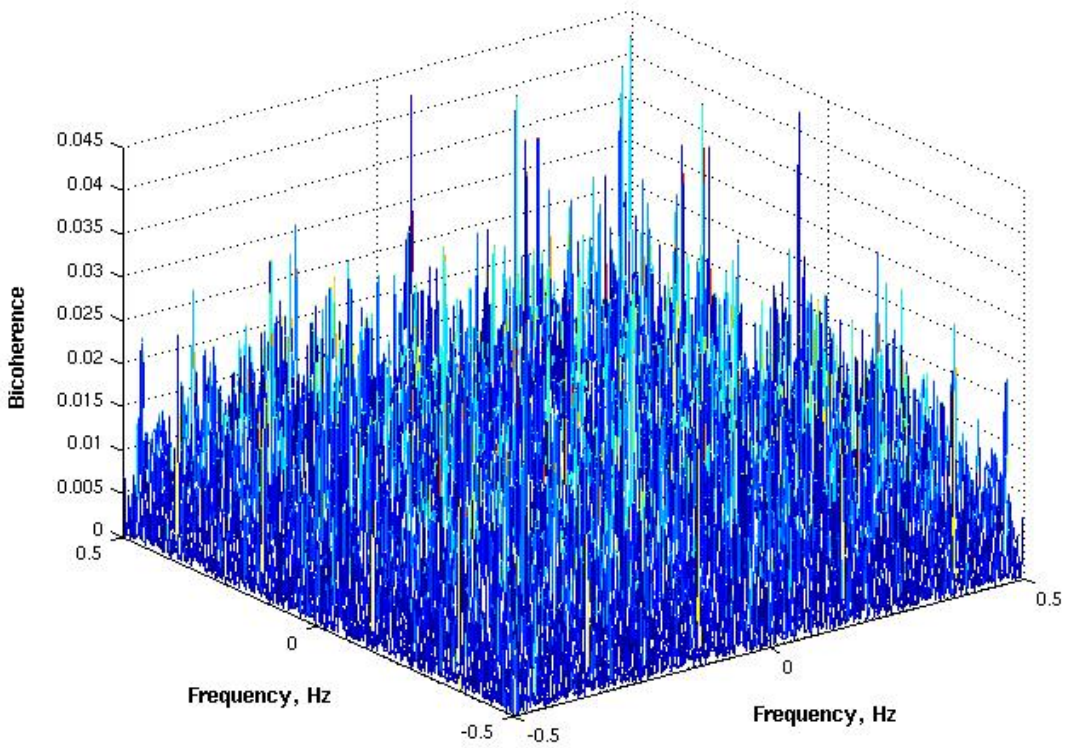

Figure 8. Bicoherence function for the linear time series. 


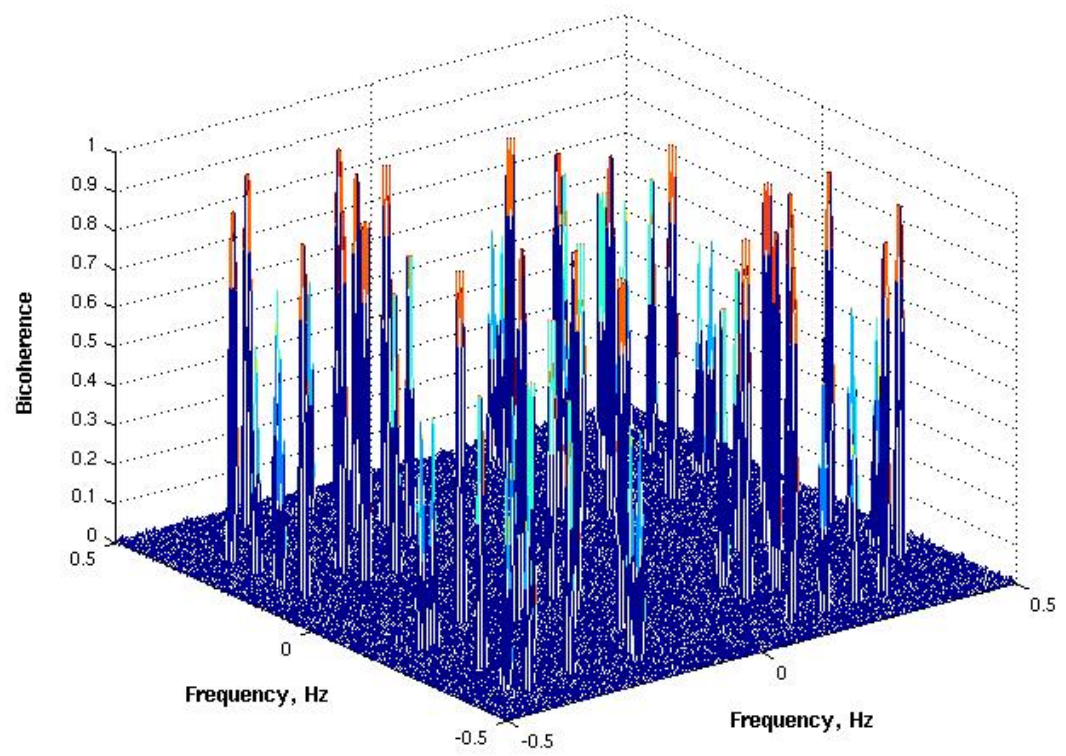

Figure 9. Bicoherence function for the nonlinear time series.

In addition, the symmetry associated with the computation of the bicoherence function is also evident in the contour plot. The bicoherence function basically computes the correlation between two frequencies and the sum of those two frequencies. Therefore, for example, the bicoherence function for the first frequency (x-axis) being $2 \mathrm{~Hz}$ and the second frequency (y-axis) being $5 \mathrm{~Hz}$ will be the same as the bicoherence for the first frequency being $5 \mathrm{~Hz}$ and the second frequency being $2 \mathrm{~Hz}$ since their sum is the same.

This symmetry is presented in figure 11. As figure 11 indicates, knowledge of Region I (two such regions in the first quadrant) and Region II (two such regions in the fourth quadrant) is sufficient to completely define the remainder of the quadrants due to symmetry considerations. Regions I and II indicate that the original frequencies have been quadratically-coupled, resulting in new frequencies that are the sum (positive and negative) of the original frequencies. The new frequencies include $0.18,0.24$, and 0.42 . The limitation of Region I to the triangle shown is due to the fact that the summation of two frequencies cannot exceed the Nyquist frequency (0.5, in this case). Therefore, the combination of the second frequency $(0.30 \mathrm{~Hz})$ with itself is not included since $0.60 \mathrm{~Hz}$ would be greater than the Nyquist frequency.

For subsequent results, although the bicoherence function is computed for all frequencies (positive and negative), Region I in the first quadrant will be of primary interest for the sake of simplicity. This will be sufficient to demonstrate the applicability and value of HOS to flight flutter data.

\section{Results}

In this section, data analysis using traditional and HOS methods is presented. The data analyzed consists of data acquired using sine dwells and lateral stick raps. The sine dwell data represents data acquired subcritically (no immediate onset of flutter or LCO). The lateral stick rap data represents data that was acquired as an LCO event was approached. The inputs (sine dwells and lateral stick raps) are in degrees of aileron motion and the outputs are accelerations at the forward launcher location (at the wing tip). In addition, computed results using the lateral stick rap data, generated using a method developed by Silva, ${ }^{5}$ are presented. Using this method, an impulse response is identified from the experimental input/output data and used to determine the level of linear/nonlinear behavior at a given condition. 


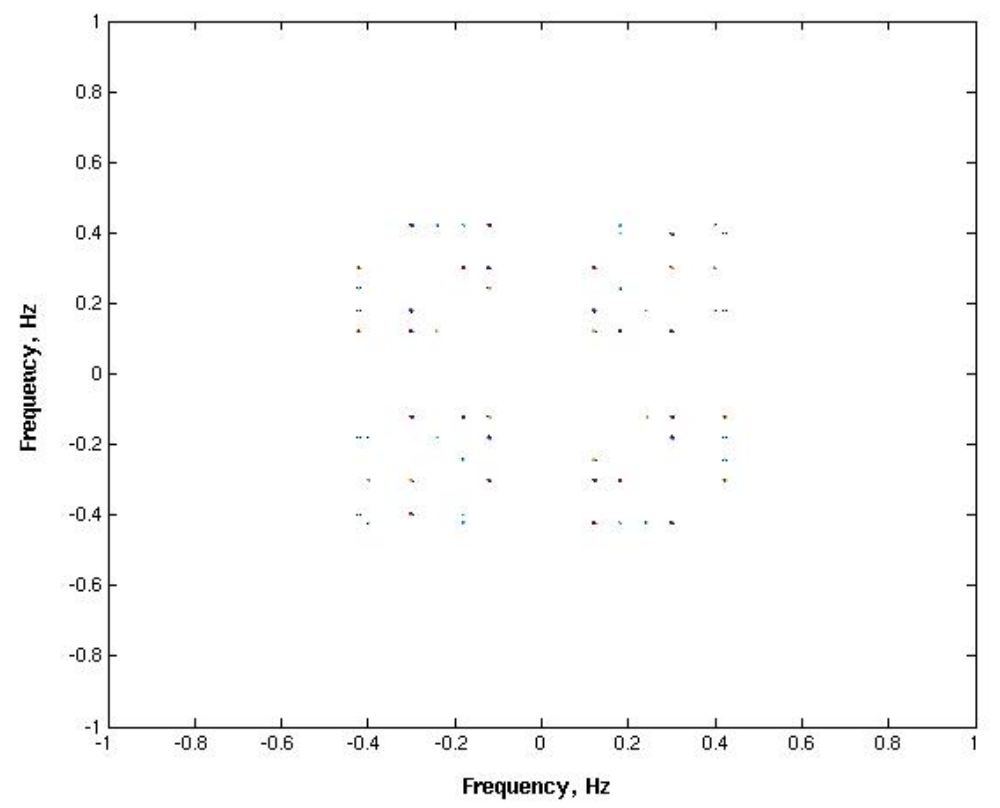

Figure 10. Contour plot of the bicoherence function for the nonlinear time series.

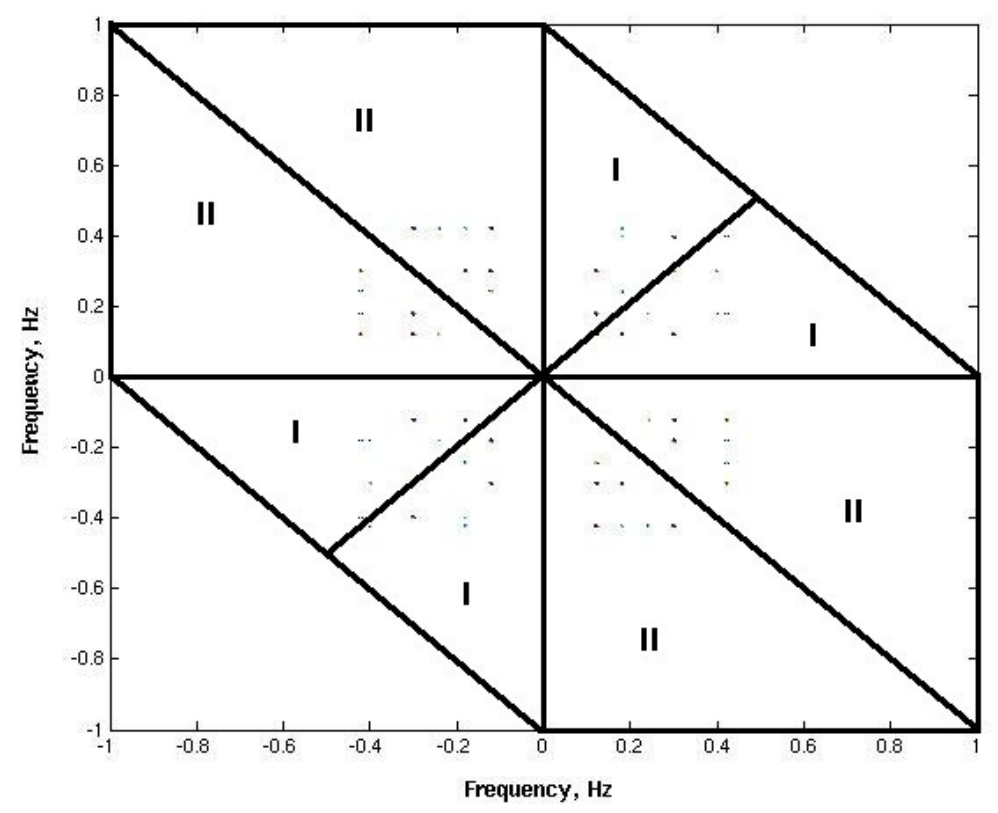

Figure 11. Contour plot of the bicoherence function for the nonlinear time series with symmetry regions identified. 


\section{A. Sine Dwell Data}

Figure 12 presents a series of sine dwell inputs applied using the ailerons and the corresponding accelerations measured at the forward tip of the starboard store at a given flight condition. These dwells were performed at specific frequencies ranging from 4.9 to $6.1 \mathrm{~Hz}$ in an attempt to excite the LCO condition. It is obvious from the acceleration responses that an LCO response was not excited at this flight condition.
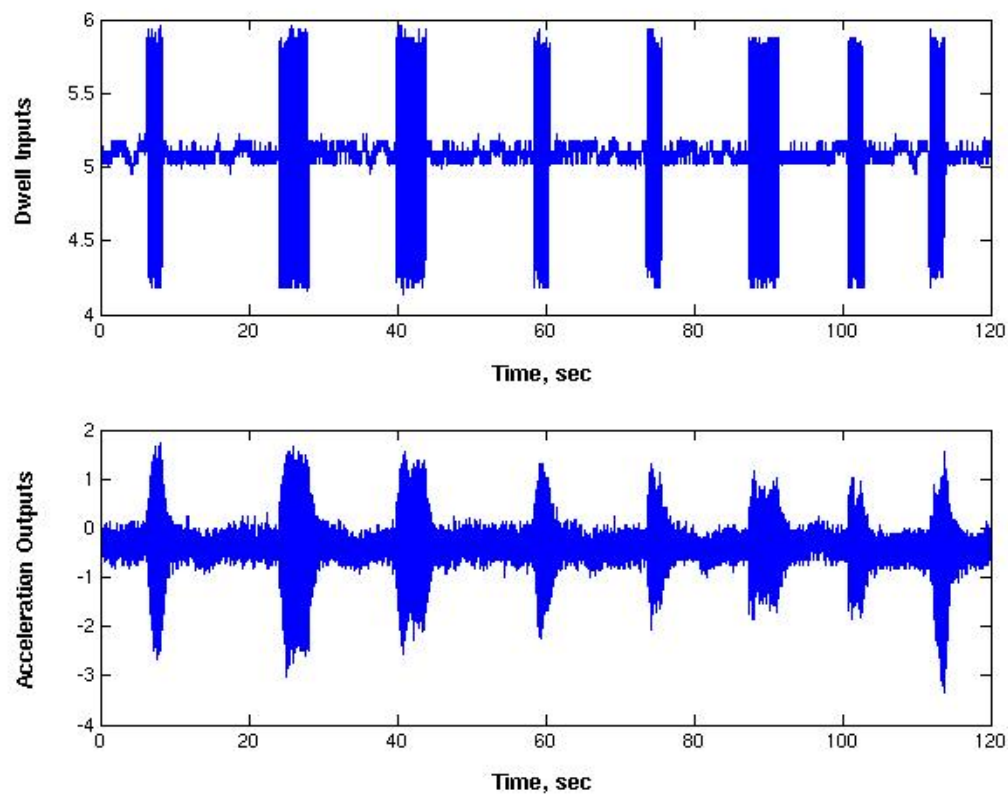

Figure 12. Sine dwells using ailerons and corresponding acceleration responses.

However, it is important to analyze the data to determine the level of nonlinear interactions (if any) that may be present. Figure 13 presents the magnitude of the frequency response for the last dwell shown in figure 12 and the magnitude of the frequency response for the corresponding acceleration. It is interesting to note that, although the input excitation is at approximately $6 \mathrm{~Hz}$, the acceleration exhibits a response at 5 $\mathrm{Hz}$ and at, approximately, $10 \mathrm{~Hz}$. Based on the fact that a purely linear response would exhibit only a $6 \mathrm{~Hz}$ peak (same as the input), this response clearly is exhibiting a nonlinear interaction. In this situation, where the input spectrum is known, the classification of a response as being linear or nonlinear is straightforward. However, in many experimental situations, only an output measurement is available, precluding this type of system response classification.

In order to better understand the nature of this nonlinear coupling, the bicoherence function is computed using the acceleration data. Presented in figure 14 is the bicoherence (or auto-bicoherence) for the acceleration data. As can be seen, there is a large spike close to unity in value, indicating that the nonlinear coupling is quadratic in nature. Viewing a contour plot of this bicoherence function and zooming in (keeping in mind the symmetry considerations for this function), we see the result presented in figure 15 . According to figure 15 , the $5 \mathrm{~Hz}$ frequency is quadratically-coupled with itself, resulting in the creation of the $10 \mathrm{~Hz}$ frequency response.

\section{B. Lateral Stick Rap Input Data}

Figure 16 presents a lateral stick rap input via the aileron and the resultant output acceleration measured at the forward launcher tip. At this condition, the aircraft does not exhibit any type of instability and basically returns to its original, undisturbed position.

The power spectrum density (PSD) plots for the lateral stick rap input and output and the linear coherence function for this input/output pair are presented as figure 17. 

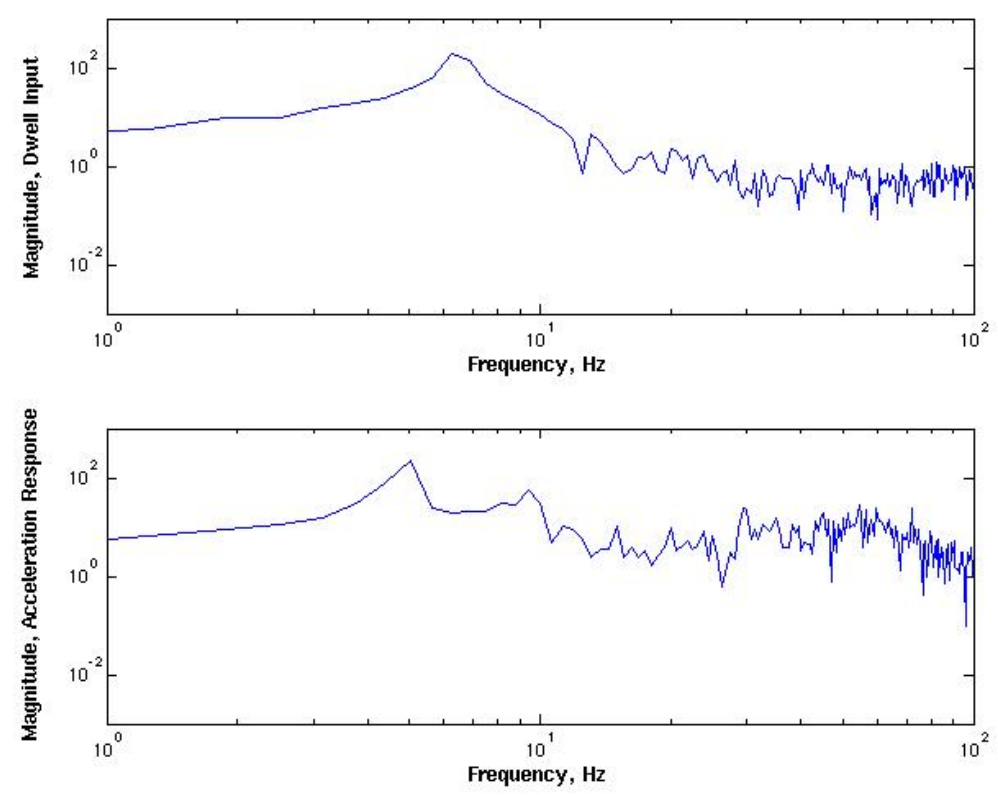

Figure 13. Magnitude of frequency response of last sine dwell from previous figure and corresponding acceleration response.

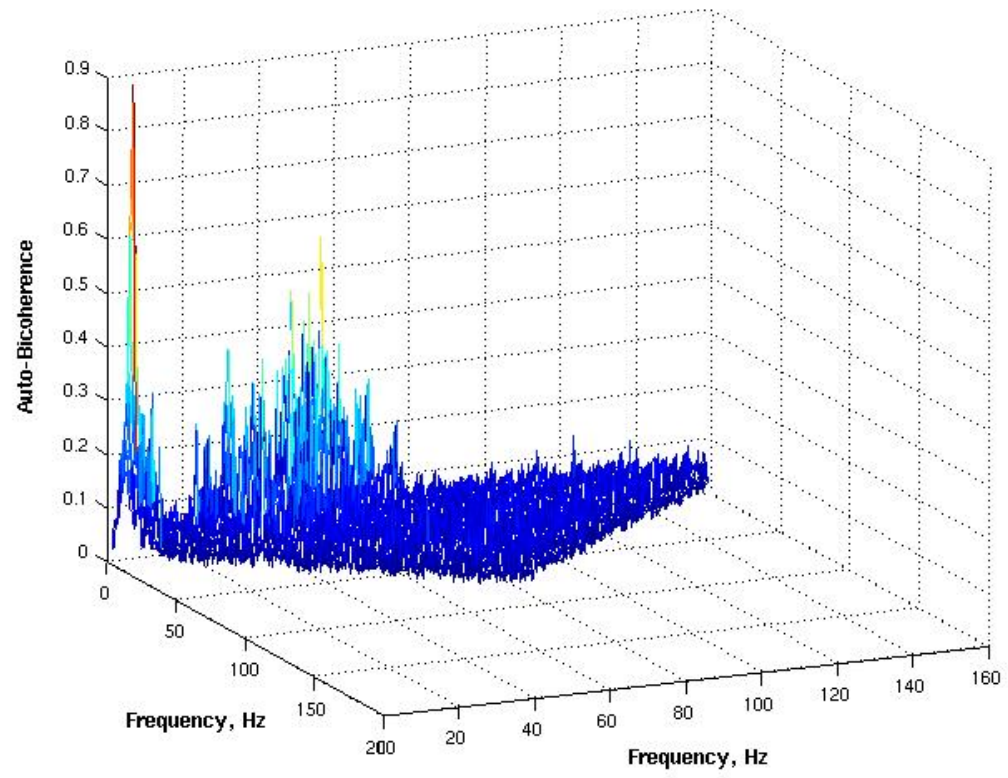

Figure 14. Bicoherence function for the acceleration data. 


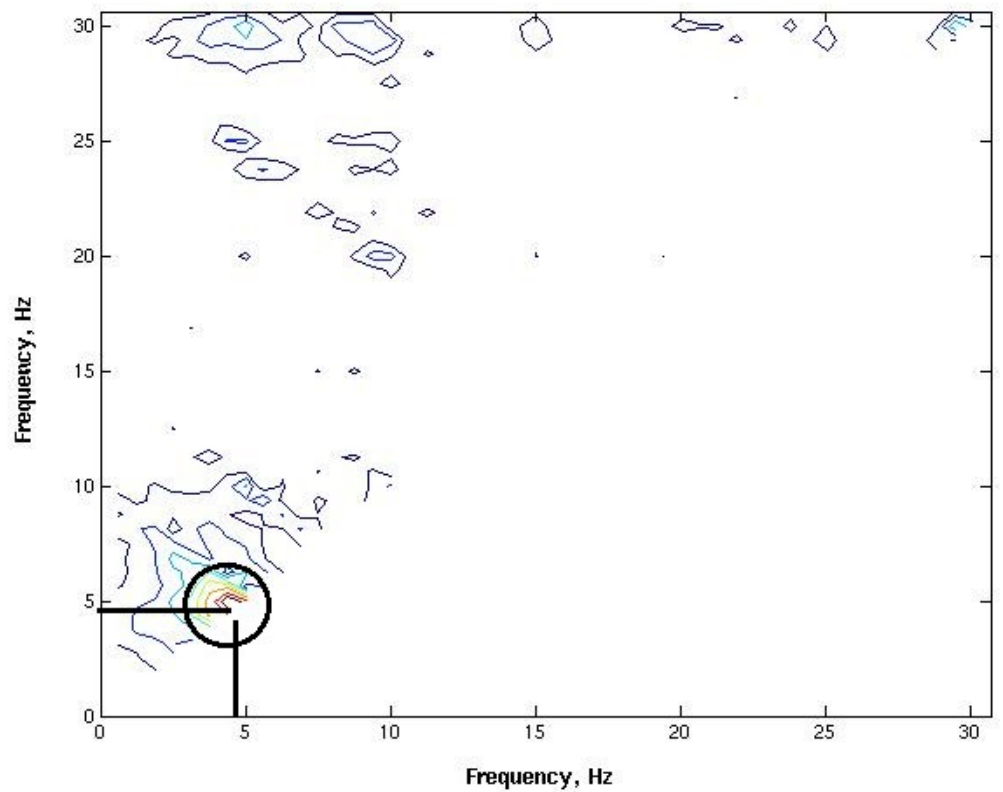

Figure 15. Contour plot of the bicoherence function for the acceleration data.
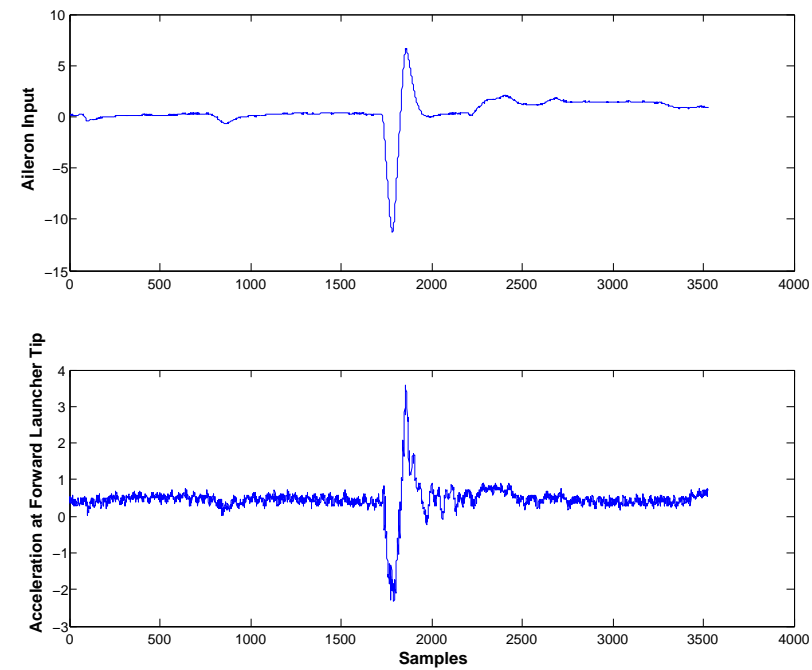

Figure 16. Lateral stick rap input and acceleration response for benign condition. 

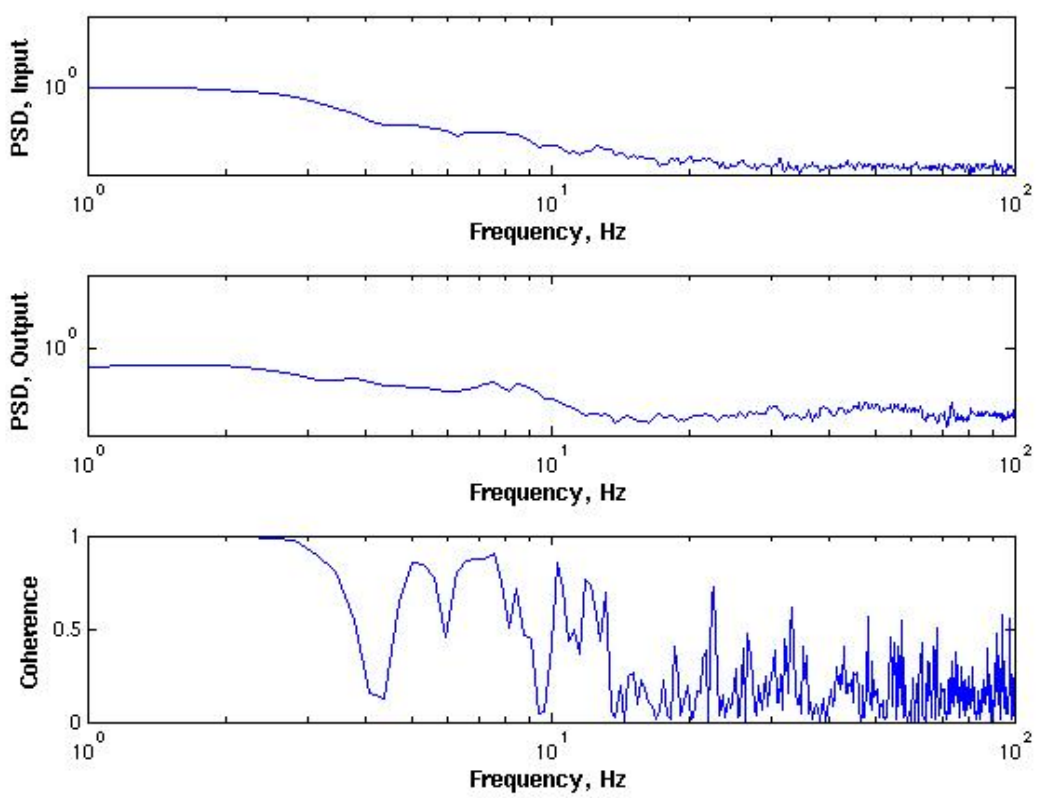

Figure 17. Power spectrum density plots for lateral stick rap input and acceleration output and linear coherence function for this input/output pair at benign flight condition.

As can be seen, the PSD of the output does not exhibit any noticeable differences from the PSD of the input, indicative of a predominantly linear situation. The linear coherence function indicates strong correlation (indicative of linear behavior) up to about $3 \mathrm{~Hz}$, with subsequent peaks falling below unity (indicative of nonlinear behavior). A broad peak at around $5 \mathrm{~Hz}$ with a coherence of about 0.8 is present but with no significant peaks at this frequency in the PSD of the input and output, it is difficult to glean additional information from this plot.

In order to provide additional guidance regarding the linear or nonlinear nature of the current flight condition, the bicoherence of the output is generated. The output can be separated into two regions: the region prior to the application of the lateral stick rap input and the region after application of the lateral stick rap input. The bicoherence for the output data prior to the lateral stick rap input is presented as figure 18. The small values of the bicoherence and the lack of any significant peaks supports the assertion that this flight condition is predominantly linear in nature.

The bicoherence for the output data after the lateral stick rap input is presented as figure 19. Although the values of the bicoherence are still low, there are some noticeable peaks that were not present in the previous figure. These peaks are in the region of the $5 \mathrm{~Hz}$ LCO phenomenon. This serves to illustrate the high sensitivity of the HOS functions to identify potential nonlinear couplings at conditions that would appear benign using traditional methods.

Shown in figure 20 is the lateral stick rap input and acceleration response for a different flight condition. At this condition, it is clear that the input has excited the aircraft into an LCO event. The PSD of the input and output and the coherence function for this condition is presented as figure 21. The PSD of the output reveals a strong peak at about $5.5 \mathrm{~Hz}$, the LCO frequency. Since the coherence function indicates a very low value for this frequency, this implies that this frequency is not linearly correlated with the input (i.e, a nonlinear response).

The bicoherence function for the output data prior to the application of the lateral stick rap for this condition is presented as figure 22. The larger values displayed by this bicoherence function supports the increasing level of nonlinear interactions that are ocurring at this flight condition. Based on this result, HOS methods may be suitable for identifying regions of nonlinear dynamics prior to the full onset of the nonlinear phenomena. It is interesting to note that the bicoherence function indicates that the approximate $8 \mathrm{~Hz}$ frequency (slightly noticeable in the PSD output plot for this condition) is the result of a nonlinear 


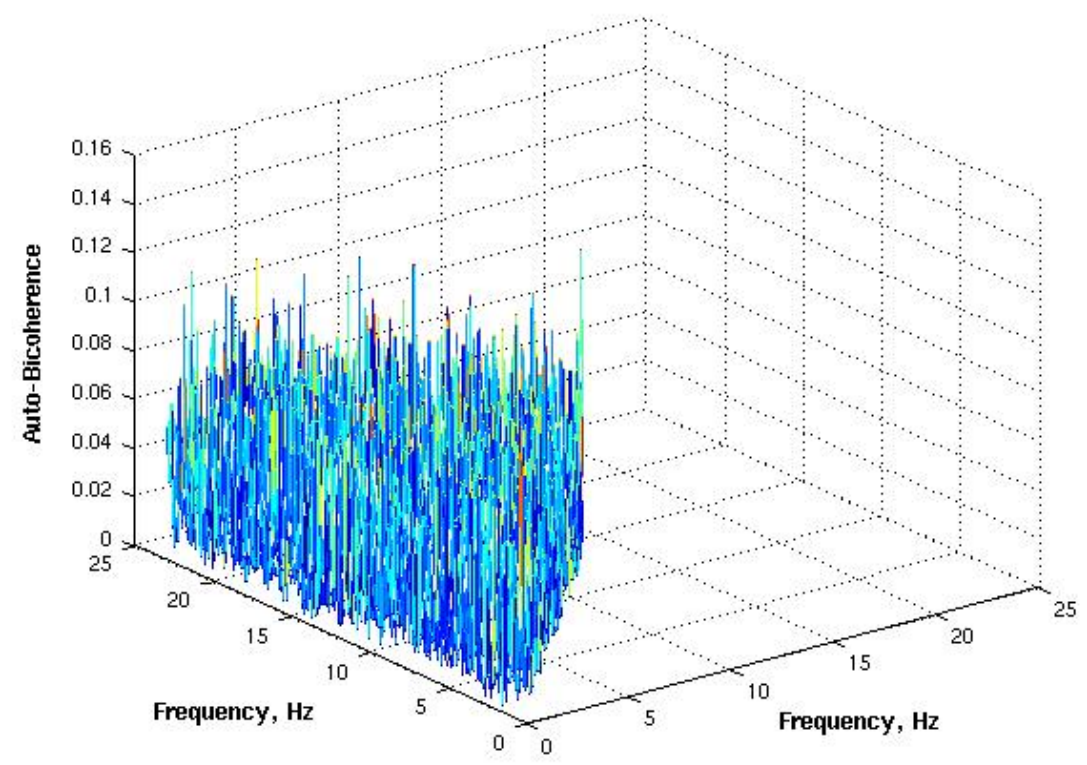

Figure 18. Bicoherence function for output data prior to lateral stick rap input at benign condition.

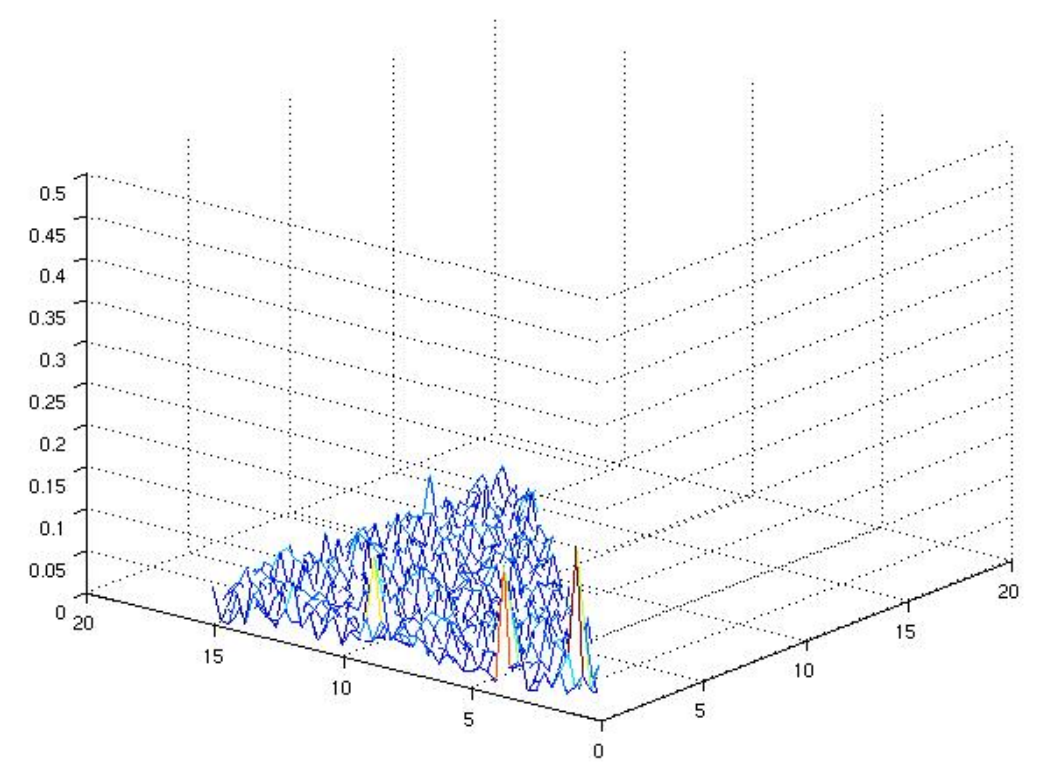

Figure 19. Bicoherence function for output data after lateral stick rap input at benign condition. 

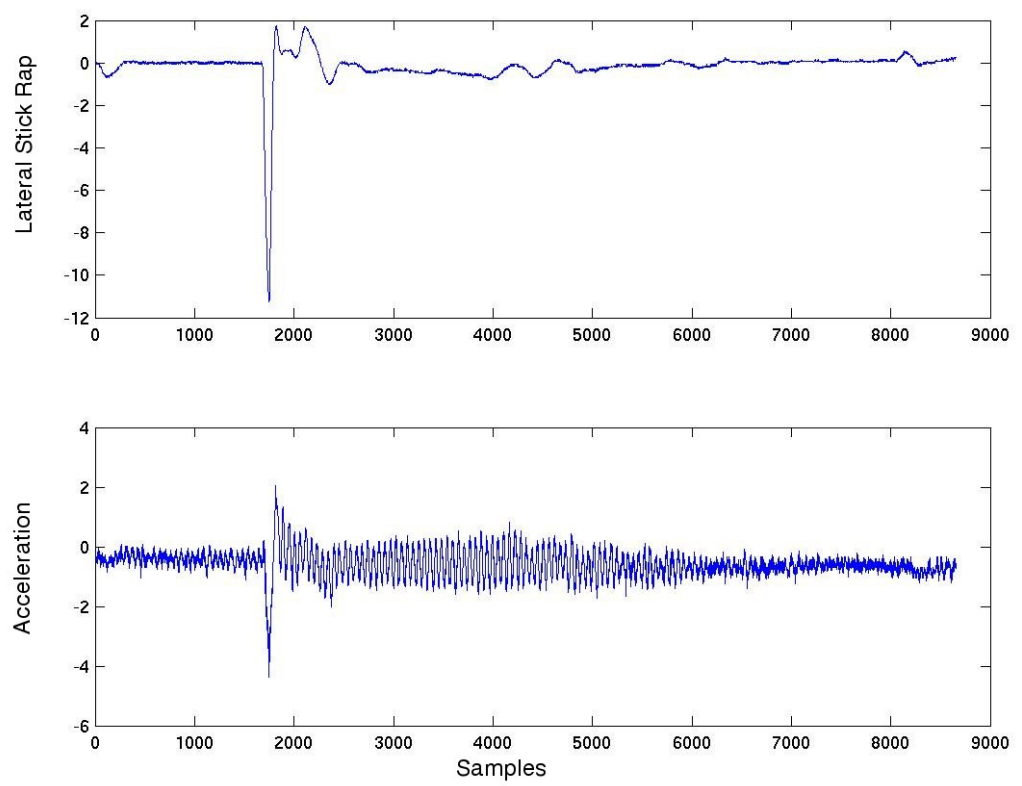

Figure 20. Lateral stick rap input and acceleration response at flight condition where an LCO is encountered.
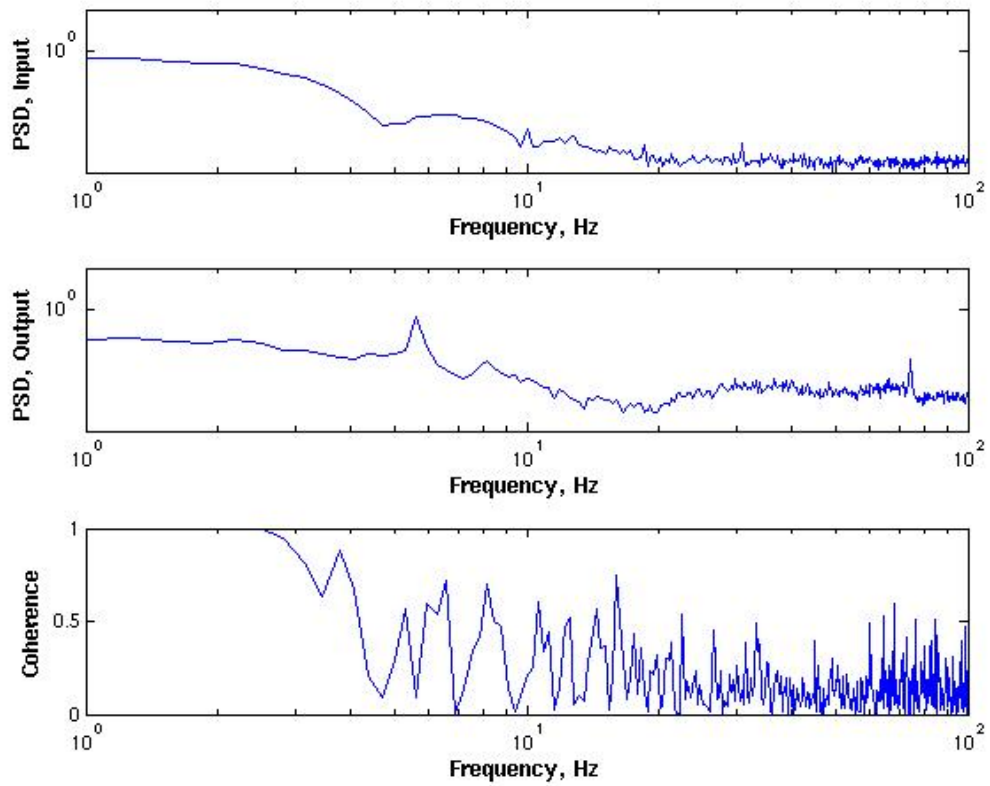

Figure 21. Power spectrum density plots for lateral stick rap input and acceleration output and linear coherence function for this input/output pair at flight condition where LCO was encountered. 
interaction.

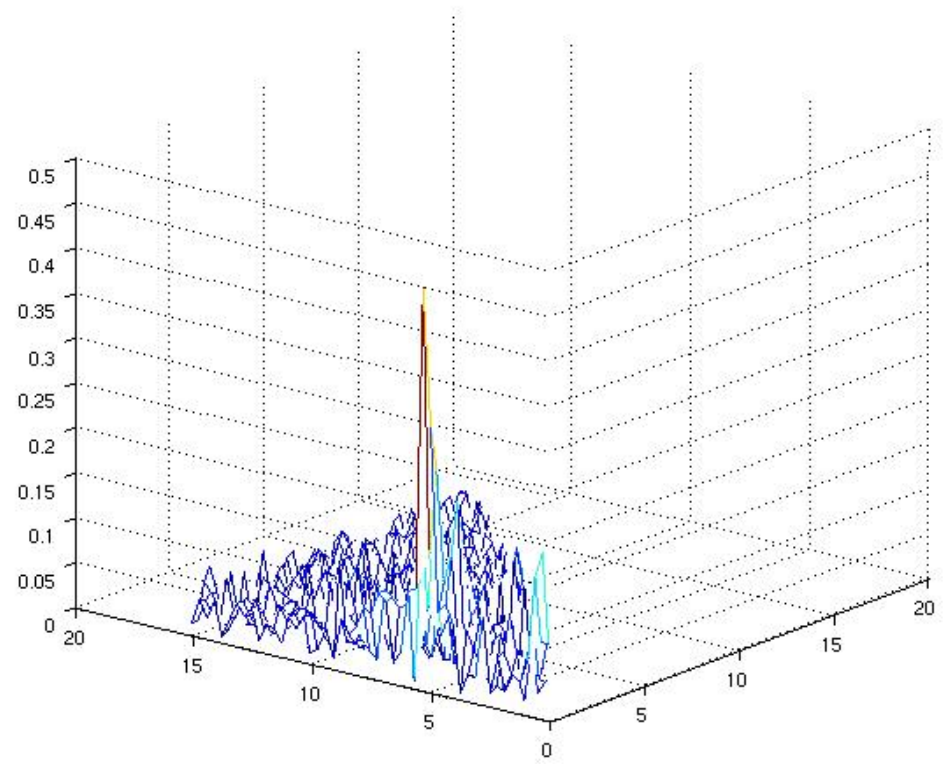

Figure 22. Bicoherence function for output data prior to lateral stick rap input at LCO condition.

Additional data analysis must be performed in order to verify the accuracy of results presented using HOS methods. Although HOS methods possess the necessary sensitivity to identify nonlinear coupling mechanisms, this sensitivity can also cause computational difficulties if caution is not exercised during computation of HOS functions.

In order to enhance the robustness of HOS methods, data manipulation techniques should be investigated to determine the optimal procedure for HOS analysis. One such potential method is briefly presented in the next section.

\section{Data Analysis Using Identified Impulse Response}

Using a method developed by Silva, ${ }^{5}$ the impulse response of the system at a given flight condition is extracted using the input (lateral stick rap) and the output (acceleration) time histories. The input, output, and identified impulse response, for a given flight condition, are presented as figure 23 .

The impulse response identified in figure 23 is then used to predict the response due to the lateral stick rap input. Since the identification procedure assumes a linear process, the response predicted using the impulse response is a linearized prediction. The level of difference between the predicted and actual (measured) responses is an indication of the presence of nonlinear effects. This comparison is presented as figure 24, where it can be seen that the difference between the two responses is not very large.

Using the same approach, the impulse response can be identified for the flight condition where an LCO phenomenon was encountered, as shown previously in figure 20. Identifying the impulse response for this flight condition and using it to predict a linearized response, the difference between the predicted and measured responses (shown in figure 25) is indicative of the highly nonlinear behavior experienced at this condition. Using the impulse response method may, therefore, also be suitable for identifying regions that are transitioning from linear to nonlinear behavior as the flight test is performed.

\section{Conclusion}

Data from RAAF F/A-18 flight flutter tests was presented and analyzed using traditional and higher-order spectral techniques. The high-quality data, which includes flutter and LCO phenomena, is well-suited for 

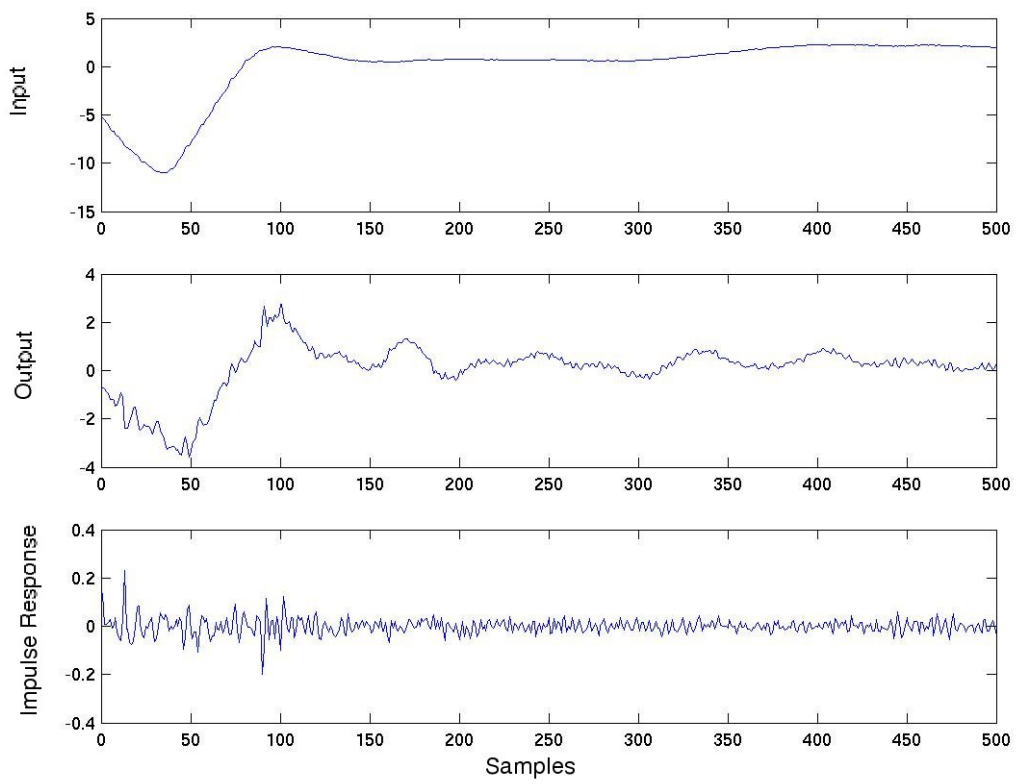

Figure 23. Lateral stick input (Input), acceleration output (Output), and impulse response identified from the input and output.

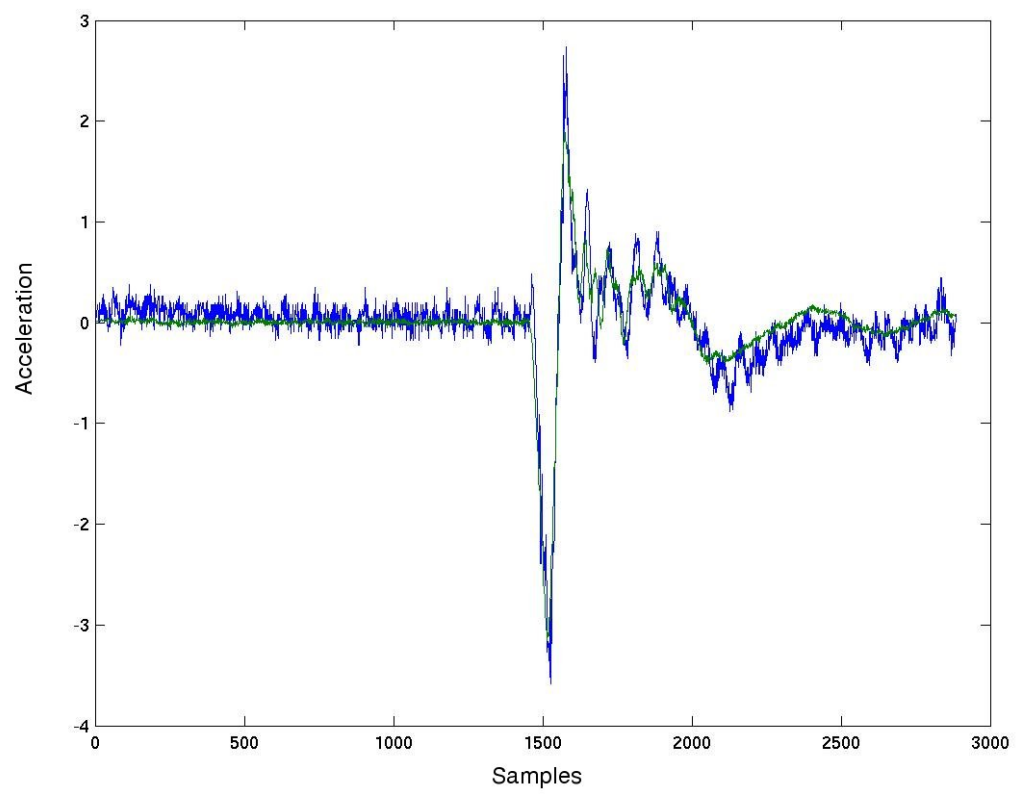

Figure 24. Comparison of predicted (green) and actual (blue) responses.

\section{8 of 20}




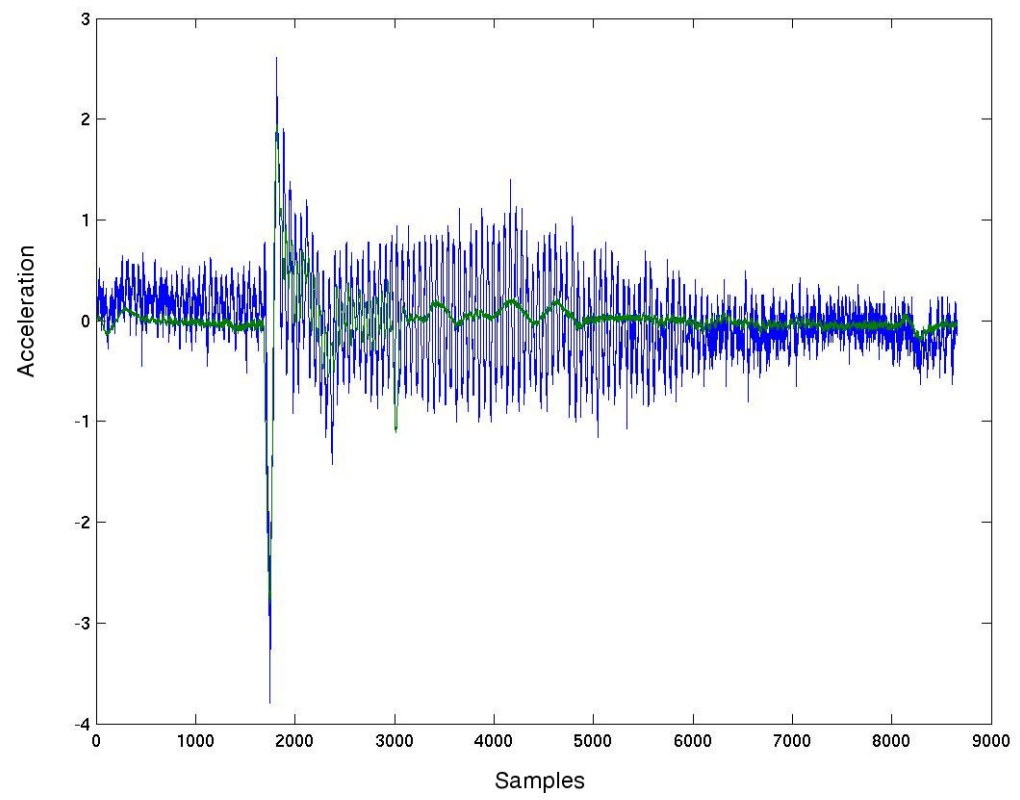

Figure 25. Comparison of predicted (green) and measured (blue) acceleration responses for flight condition where LCO was encountered.

the application of higher-order spectral methods that can be used to detect nonlinearities in experimental measurements. A method for identifying impulse responses from measured data was applied to the data and used to identify regions of linear and nonlinear behavior. Higher-order spectral analyses, primarily bicoherence functions, were generated at different flight conditions in order to understand the transition from linear to nonlinear aeroelastic behavior, including LCO phenomena. Additional research in underway to perform additional HOS data analyses in order to determine a preferred process for the routine application of HOS methods to flight test data.

\section{Acknowledgments}

The first author would like to acknowledge the support of NASA Langley's Office of Education and its support of the first author's research via the Floyd L. Thompson Fellowship. In addition, the support and education provided by Prof. Hajj (Virginia Tech) and Profs. Powers and Stearman (U. of Texas, Austin) during the first author's Fellowship is greatly acknowledged.

\section{References}

${ }^{1}$ Dowell, E. H., Edwards, J. W., and Strganac, T., "Nonlinear Aeroelasticity," Journal of Aircraft, Vol. 40, Sept. 2003, pp. 857-874.

${ }^{2}$ Dunn, S. A., Farrell, P. A., Budd, P. J., Arms, P. B., Hardie, C. A., and Rendo, C. J., "F/A-18A Flight Flutter TestingLimit Cycle Oscillation or Flutter?" International Forum on Aeroelasticity and Structural Dynamics, No. 00-2401, Madrid, Spain, June 2001.

${ }^{3}$ Trame, L. W., Williams, L. E., and Yurkovich, R. N., "Active Aeroelastic Oscillation Control on the F/A-18 Aircraft," AIAA Guidance Navigation and Control Conference, Snowmass, CO, Aug. 1985.

${ }^{4}$ Denegri, C. M., "Limit Cycle Oscillation Flight Test Results of a Fighter with External Stores," Journal of Aircraft, Vol. 37, June 2001, pp. 761-769.

${ }^{5}$ Silva, W. A., Piatak, D. J., and Scott, R. C., "Identification of Experimental Unsteady Aerodynamic Impulse Responses," Proceedings of the 44th Structures, Structural Dynamics and Materials Conference, No. 03-1959, Norfolk, VA, April 2003.

${ }^{6}$ Hajj, M. R. and Silva, W. A., "Nonlinear Flutter Aspects of the Flexible HSCT Semispan Model," Proceedings of the 44th Structures, Structural Dynamics and Materials Conference, No. 2003-1515, Norfolk, VA, April 2003.

${ }^{7}$ Hajj, M. R. and Silva, W. A., "Nonlinear Flutter Aspects of the Flexible High-Speed Civil Transport Semispan Model," 
Journal of Aircraft, Vol. 41, 2004, pp. 1202-1208.

${ }^{8}$ Prazenica, R. J., Brenner, M. J., and Lind, R., "Nonlinear Volterra Kernel Identification for the F-18 Active Aeroelastic Wing," International Forum on Aeroelasticity and Structural Dynamics, No. US-32, Amsterdam, The Netherlands, 4-6 June 2003.

${ }^{9}$ Brenner, M. J. and Prazenica, R. J., "Aeroservoelastic Modeling and Test Data Analysis of the F-18 Active Aeroelastic Wing," International Forum on Aeroelasticity and Structural Dynamics, No. US-6, Amsterdam, The Netherlands, 4-6 June 2003.

${ }^{10}$ Lind, R. and Mortagua, J. P., "Extracting Modal Dynamics from Volterra Kernels to Reduce Conservatism in the Flutterometer," International Forum on Aeroelasticity and Structural Dynamics, No. US-23, Amsterdam, The Netherlands, 4-6 June 2003.

${ }^{11}$ Arms, P., Farrell, P., and Dunn, S., "AF/A-18 ASRAAM Flutter Flight Testing - The Aussie Approach," Society of Experimental Test Pilots Symposium, Los Angeles, CA, Sept. 2000.

${ }^{12}$ Keeler, A., Arms, P., Farrell, P., and Dunn, S., "AF/A-18 / AIM-132 ASRAAM and AIM-9 Sidewinder Flutter Testing," Aircraft Stores Compatibility Symposium, Florida, March 2001.

${ }^{13}$ Juang, J.-N., Cooper, J. E., and Wright, J. R., "An Eigensystem Realization Algorithm Using Data Correlations (ERA/DC) for Modal Parameter Identification," Control Theory and Advanced Technology, Vol. 4, 1988.

${ }^{14}$ Cole, H. A., "On-the-Line Analysis of Random Vibrations," Society of Experimental Test Pilots Symposium, No. 68-288, 1968.

${ }^{15}$ Ljung, L., System Identification: Theory for the User, Prentice-Hall, Englewood Cliffs, 1987.

${ }^{16}$ Zimmerman, N. H. and Weissenburger, J. T., "Prediction of Flutter Onset Speed Based on Flight Testing at Subcritical Speeds," Journal of Aircraft, Vol. 1, 1964.

${ }^{17}$ Silva, W. A., "Identification of Nonlinear Aeroelastic Systems Based on the Volterra Theory: Progress and Opportunities," Journal of Nonlinear Dynamics, Vol. 39, Jan. 2005.

${ }^{18}$ Marzocca, P., Librescu, L., and Silva, W. A., "Nonlinear Stability and Response of Lifting Surfaces via Volterra Series," presented at the 20th International Congress of Theoretical and Applied Mechanics, Chicago, IL, 27 August - 2 September 2000.

${ }^{19}$ Marzocca, P., Librescu, L., and Silva, W. A., "Volterra Series Approach for Nonlinear Aeroelastic Response of 2-D Lifting Surfaces," presented at the 42nd Structures, Structural Dynamics, and Materials Conference, 16-19 April 2001, Seattle, WA, 16-19 April 2001.

${ }^{20}$ Kurdila, A. J., Carrol, B., Nishida, T., and Sheplak, M., "Reduced-Order Modeling for Low Reynolds Number Flow Control," SPIE Conference on Mathematics and Control in Smart Structures, Newport Beach, CA, Vol. 3667, March 1999, pp. 68-79.

${ }^{21}$ Prazenica, R., Kurdila, A., and Silva, W. A., "Multiresolution Methods for Representation of Volterra Series and Dynamical Systems," AIAA Paper 2000-1754, April 2000.

${ }^{22}$ Hajj, M. R. and Silva, W. A., "Nonlinear Flutter Aspects of the Flexible HSCT Semispan Model," International Forum on Aeroelasticity and Structural Dynamics, No. US-39, Amsterdam, The Netherlands, 4-6 June 2003.

${ }^{23} \mathrm{Kim}$, Y. C. and Powers, E. J., "Digital Bispectral Analysis and its Applications to Nonlinear Wave Interactions," IEEE Transactions of Plasma Science, Vol. PS-7, 1979, pp. 120-131.

${ }^{24}$ Hajj, M. R., Miksad, R. W., and Powers, E. J., "Fundamental Subharmonic Interaction: Effect of the Phase Relation," Journal of Fluid Mechanics, 1993.

${ }^{25}$ Hajj, M. R., Miksad, R. W., and Powers, E. J., "Perspective: Measurements and Analyses of Nonlinear Wave Interactions with Higher-Order Spectral Moments," Journal of Fluid Engineering, 1997.

${ }^{26}$ Choudhury, M. A. A. S., Shah, S. L., and Thornhill, N. F., "Detection and Diagnosis of System Nonlinearities Using Higher Order Statistics," 15th Triennial IFAC World Congress, Barcelona, Spain, 2002. 